\title{
Effects of Fingolimod and Natalizumab on Brain T1-/T2-Weighted and Magnetization Transfer Ratios: a 2-Year Study
}

\author{
Paolo Preziosa ${ }^{1,2} \cdot$ Loredana Storelli $^{1}$ - Alessandro Meani ${ }^{1} \cdot$ Lucia Moiola $^{2} \cdot$ Mariaemma Rodegher $^{2}$. \\ Massimo Filippi ${ }^{1,2,3,4,5} \cdot$ Maria A. Rocca ${ }^{1,2,4}$
}

Accepted: 18 December 2020 / Published online: 22 January 2021

(C) The American Society for Experimental NeuroTherapeutics, Inc. 2021

\begin{abstract}
Fingolimod and natalizumab significantly reduce disease activity in relapsing-remitting multiple sclerosis (RRMS) and could promote tissue repair and neuroprotection. The ratio between conventional T1- and T2-weighted sequences (T1w/T2w-ratio) and magnetization transfer ratio (MTR) allow to quantify brain microstructural tissue abnormalities. Here, we compared fingolimod and natalizumab effects on brain T1w/T2w-ratio and MTR in RRMS over 2 years of treatment. RRMS patients starting fingolimod $(n=25)$ or natalizumab $(n=30)$ underwent 3 T brain MRI scans at baseline (T0), month 6 (M6), month 12 (M12), and month 24 (M24). White matter (WM) lesions, normal-appearing (NA) WM, and gray matter (GM) T1w/T2w-ratio and MTR were estimated and compared between groups using linear mixed models. No baseline demographic, clinical, and MRI difference was found between groups. In natalizumab patients, lesion T1w/T2w-ratio and MTR significantly increased at M6 vs. T0 ( $p \leq$ $0.035)$ and decreased at subsequent timepoints $(p \leq 0.037)$. In fingolimod patients, lesion T1w/T2w-ratio increased at M12 vs. T0 $(p=0.010)$, while MTR gradually increased at subsequent timepoints vs. T0 $(p \leq 0.027)$. Natalizumab stabilized NAWM and GM T1w/T2w-ratio and MTR. In fingolimod patients, NAWM T1w/T2w-ratio and MTR significantly increased at M24 vs. M12 $(p \leq 0.001)$. A significant GM T1w/T2w-ratio decrease at M6 vs. T0 $(p=0.014)$ and increase at M24 vs. M6 $(p=0.008)$ occurred, whereas GM MTR was significantly higher at M24 vs. previous timepoints $(p \leq 0.017)$ with significant betweengroup differences $(p \leq 0.034)$. Natalizumab may promote an early recovery of lesional damage and prevent microstructural damage accumulation in NAWM and GM during the first 2 years of treatment. Fingolimod enhances tissue damage recovery being visible after 6 months in lesions and after 2 years in NAWM and GM.
\end{abstract}

Key Words Multiple sclerosis $\cdot$ MRI $\cdot$ disease-modifying drugs $\cdot$ magnetization transfer ratio $\cdot \mathrm{T} 1 \mathrm{w} / \mathrm{T} 2 \mathrm{w}$-ratio $\cdot$ neuroprotection $\cdot$ repair

Maria A. Rocca

rocca.mara@hsr.it

1 Neuroimaging Research Unit, Division of Neuroscience, IRCCS San Raffaele Scientific Institute, Milan, Italy

2 Neurology Unit, IRCCS San Raffaele Scientific Institute, Milan, Italy

3 Neurophysiology Service, IRCCS San Raffaele Scientific Institute, Milan, Italy

4 Vita-Salute San Raffaele University, Via Olgettina, 60, 20132 Milan, Italy

5 Neurorehabilitation Unit, IRCCS San Raffaele Scientific Institute, Milan, Italy

\section{Introduction}

The paradigm of multiple sclerosis (MS) treatment is experiencing a dramatic evolution. The notion that effective disease-modifying drugs (DMDs) should be started early to limit clinical and magnetic resonance imaging (MRI) disease activity and disability progression is combined with the recognized relevance of promoting neuroprotection and repair.

Accordingly, besides the effects in preventing clinical relapses and new MRI lesions, there is the urgent need to explore the potential neuroprotective effects of available DMDs. Thanks to its sensitivity and specificity to the different pathological substrates of MS, MRI offers the possibility to investigate these beneficial effects of treatments in vivo [1].

Magnetization transfer ratio (MTR) and the ratio between conventional T1-weighted (T1w) and T2-weighted (T2w) 
sequences (i.e., T1w/T2w-ratio) have been proposed as reliable measures to evaluate brain microarchitecture, easily implementable in the clinical setting. Since MTR provides an estimate of the extent of tissue integrity prominently associated with myelin content [2-4], it has been already applied to investigate treatment effects [5]. By assessing the dynamics occurring after active lesion formation, several studies have consistently demonstrated that different drugs and hematopoietic stem cell transplantation promote a recovery of MTR values after the acute phase [5-8]. As also supported by a post-mortem MRI-histopathology study on a white matter (WM) lesion, this is likely to reflect recovery of lesional tissue damage and remyelination [9]. Additionally, a subset of randomized controlled trials and observational studies have suggested that, by preventing longitudinal decline of MTR values, several DMDs could protect against the accumulation of microscopic damage in the whole brain, gray matter (GM), and WM [5, 6, 10-12].

$\mathrm{T} 1 \mathrm{w} / \mathrm{T} 2 \mathrm{w}$-ratio has been proposed to enhance sensitivity and specificity for myelin involvement, but also dendrite density $[13,14]$. However, the effect of treatment on this semi-quantitative measure has not been investigated yet.

Fingolimod and natalizumab are highly effective drugs for RRMS able to significantly limit clinical and MRI disease activity, with natalizumab being slightly superior to fingolimod [15]. A single-center MTR study showed that, compared to interferon beta-1a, natalizumab significantly promoted MTR recovery and prevented the further accumulation of microstructural tissue abnormalities in both focal WM lesions and normal-appearing (NA) brain tissues [6]. In progressive MS patients, natalizumab treatment for 60 weeks was associated with a significant increase of GM MTR and NAWM MTR and fractional anisotropy [10]. Using diffusion tensor MRI, three separate studies suggested that fingolimod [16] and natalizumab [17] could reduce the extent and severity of NAWM damage after 1 year and stabilize NAWM damage for 4 years [18]. In another study, natalizumab promoted tissue recovery in WM lesions but was associated with a progressive decrease of fractional anisotropy and axial diffusivity in NAWM over 1 year [19]. As a matter of fact, it remains unclear whether these two drugs could exert similar neuroprotective effects and promote tissue repair.

In two previous studies on the same cohorts analyzed here, we demonstrated that both drugs can reduce disease activity and improve cognition in RRMS, with a superiority of natalizumab in limiting lesion accumulation [20]. Moreover, these drugs were found to exert neuroprotective effects and to be associated with heterogeneous patterns of GM and WM atrophy progression, thus suggesting that different brain regions may be differently influenced by the beneficial effects of these drugs [21].
However, brain atrophy is an irreversible end-stage phenomenon; thus, the evaluation of other MRI measures able to identify neurodegenerative but also neuroreparative mechanisms of specific drugs, possibly before the occurrence of irreversible tissue loss, could be a rewarding strategy to better understand the beneficial effects of natalizumab and fingolimod.

By evaluating the effects of fingolimod and natalizumab on MTR and T1w/T2-w ratio measures in different brain compartments (i.e., lesions, NAWM, and GM), we compared the dynamic patterns of microstructural tissue features occurring with these treatments and explored their effects in preventing microstructural tissue damage progression and promoting tissue repair over 2 years.

\section{Methods}

\section{Study Design}

This was a single-center, prospective, longitudinal, open-label, non-randomized study, as previously described [20, 21]. Figure 1 summarizes the main inclusion and exclusion criteria and study flowchart. Twenty-five RRMS patients treated with fingolimod and 30 treated with natalizumab completed the study and were included in the analysis. Clinical and MRI evaluation were performed at baseline (T0), month 6 (M6), month 12 (M12), and month 24 (M24). Approval was received from the local ethical standards committee, and written informed consent was obtained from all study participants prior to enrollment.

\section{Clinical Evaluation}

At each visit, the Expanded Disability Status Scale (EDSS) score, relapses, and annualized relapse rate (ARR) were rated by a neurologist unaware of the MRI results. At T0, treatment history and relapses during the year before treatment initiation were also recorded. At follow-up, confirmed disability progression (CDP) was defined when EDSS score increased $\geq$ 1.0 point if baseline EDSS score was $\geq 1.0$, or $\geq 1.5$ points if baseline score was $0[22,23]$, and sustained for 3 months.

\section{MRI Acquisition}

Using a 3.0-Tesla scanner (Intera, Philips Medical Systems, Best, The Netherlands) under a regular maintenance program, the following brain images were acquired from all participants at each timepoint: (a) dual-echo turbo spin-echo (repetition time $[\mathrm{TR}] /$ echo time $[\mathrm{TE}]=2599 / 16,80 \mathrm{~ms}$, echo train length $[\mathrm{ETL}]=6$; flip angle $[\mathrm{FA}]=90^{\circ}$, matrix size $=256 \times 256$, field of view $[\mathrm{FOV}]=240 \times 240 \mathrm{~mm}^{2}, 44$ contiguous, $3 \mathrm{~mm}$-thick axial slices); (b) three-dimensional (3D) $T_{1^{-}}$ 


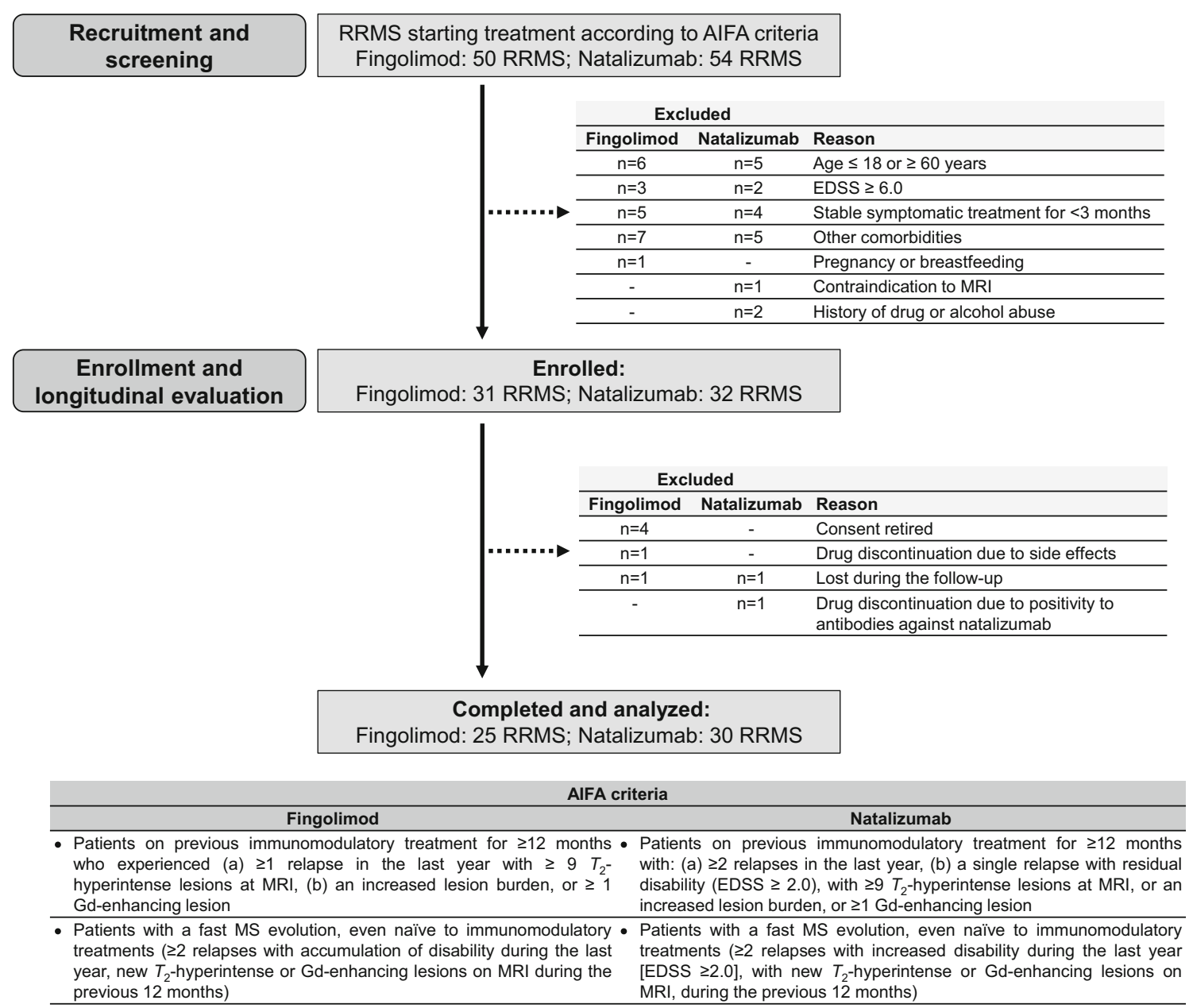

Fig. 1 Study flow diagram. Abbreviations: AIFA = Agenzia Italiana del Farmaco (Italian Medicine Agency); EDSS = Expanded Disability Status Scale; $\mathrm{Gd}=$ gadolinium; $\mathrm{MRI}=$ magnetic resonance imaging; $\mathrm{RRMS}=$ relapsing-remitting multiple sclerosis

weighted fast field echo (FFE) $\left(\mathrm{TR} / \mathrm{TE}=25 / 4.6 \mathrm{~ms} ; \mathrm{FA}=30^{\circ}\right.$; matrix size $=256 \times 256 ; \mathrm{FOV}=230 \times 230 \mathrm{~mm}^{2} ; 220$ contiguous axial slice, voxel size $=0.89 \times 0.89 \times 0.8 \mathrm{~mm}$ ); (c) $3 \mathrm{D}$ $T_{1}$-weighted FFE with and without off-resonance saturation pulses applied $\left(\mathrm{TR} / \mathrm{TE}=66 / 3.8 \mathrm{~ms}\right.$; flip angle $=18^{\circ}$; matrix size $=224 \times 224 ; \mathrm{FOV}=224 \mathrm{~mm} \times 168 \mathrm{~mm} ; 30$ contiguous axial slices with voxel size $=1 \times 1 \times 4 \mathrm{~mm}$; magnetization transfer pulse: frequency offset $=1.1 \mathrm{kHz}$, bandwidth $=$ $300 \mathrm{~Hz}$, flip angle $\left.620^{\circ}\right)$; (d) post-contrast $(0.1 \mathrm{mmol} / \mathrm{kg}$ of Gd-DTPA; acquisition delay: $5 \mathrm{~min}$ ) $T_{1}$-weighted inversion recovery sequence $(\mathrm{TR} / \mathrm{TE} /$ inversion time $[\mathrm{TI}]=2000 / 10 /$ $800 \mathrm{~ms}, \mathrm{ETL}=5 ; \mathrm{FA}=90^{\circ}$, matrix size $=400 \times 320, \mathrm{FOV}=$ $230 \mathrm{~mm} \times 195.5 \mathrm{~mm}, 44$ contiguous, 3 -mm-thick axial slices). For all scans, the slices were positioned parallel to a line joining the most infero-anterior and infero-posterior margins of the corpus callosum, with careful repositioning at follow-up.

\section{Conventional MRI Analysis}

At each visit, T2-hyperintense and Gd-enhancing lesion volumes (LVs) were measured using a local thresholding segmentation technique (Jim 6.0 software, www.xinapse. com) by consensus of two observers blinded to subjects' data. At follow-up, the number of Gd-enhancing lesions and new T2-hyperintense lesions were counted.

WM lesion masks were created by binarizing the segmentation of T2-hyperintense lesions previously obtained.

Masks of GM, WM, and cerebrospinal fluid were obtained on 3D T1-weighted images using the three-tissue type segmentation from the SPM toolbox (v12, Matlab $\left.{ }^{\circledR}\right)$ after applying lesion filling [24]. NAWM was then obtained by removing WM lesions from WM mask.

\section{T1w/T2w-Ratio Quantification}

$\mathrm{T} 1 \mathrm{w} / \mathrm{T} 2-\mathrm{w}$ ratio was obtained with an in-house pipeline adapted from Ganzetti et al. [25] and implemented in Matlab ${ }^{\circledR}$ (v12). In details, native T1- and T2-weighted sequences underwent a first step consisting of intensity bias correction (unbiased images) using N4ITK algorithm [26]. Then, unbiased images were "calibrated" adjusting the intensity histograms according to the lowest and the highest 
intensity peaks derived from the ocular and temporal muscles masks on both T1- and T2-weighted images (calibrated images). T2-weighted sequences were then co- registered on T1-weighted images and the ratio between these images (i.e., T1w/T2w-ratio) was finally calculated (Fig. 2). a

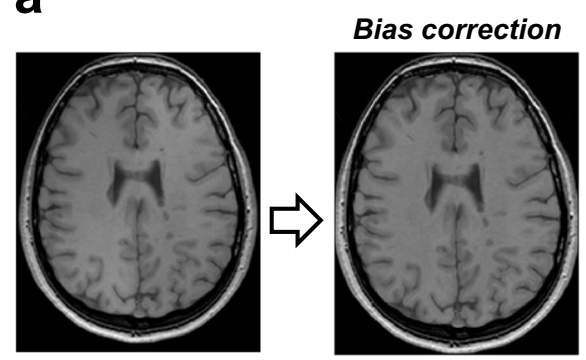

Bias correction

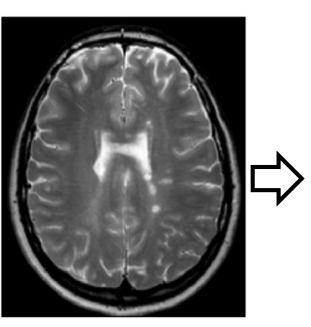

b $\quad M_{0}$

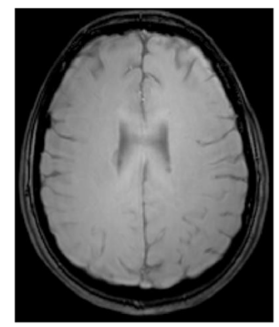

C
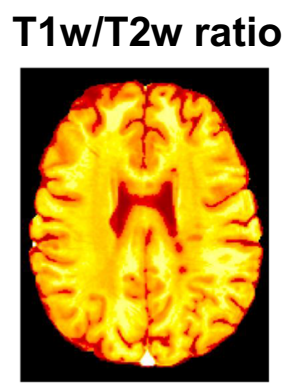

0

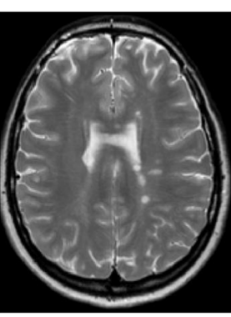

$\Rightarrow$
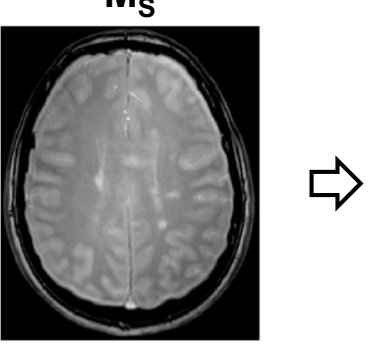

\section{MTR}

3D T1w

$\Rightarrow$

T2w

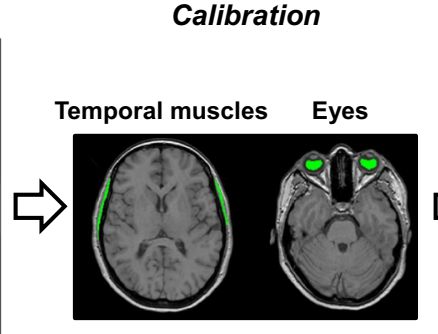

Calibratio
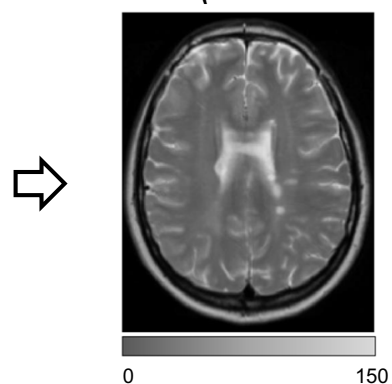
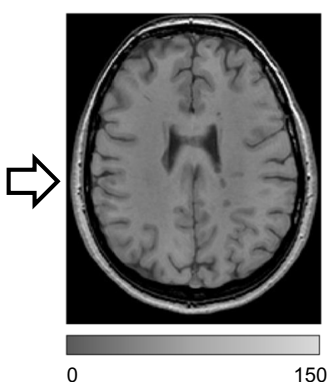

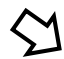

$\mathrm{T} 1 \mathrm{w} / \mathrm{T} 2 \mathrm{w}$ ratio
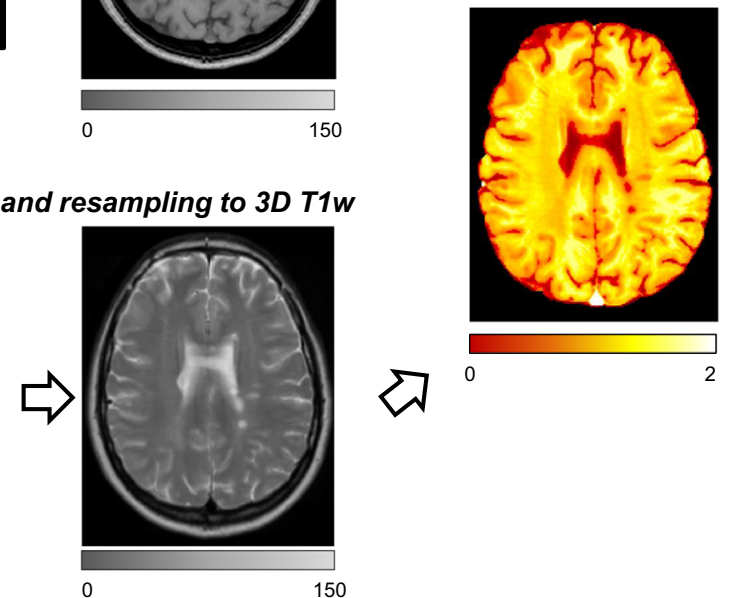

0

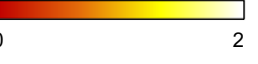

$\left.\left[\left(M_{0}-M_{s}\right) / M_{0}\right] * 100\right)$

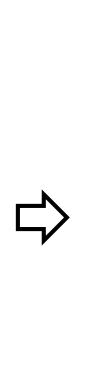

MTR
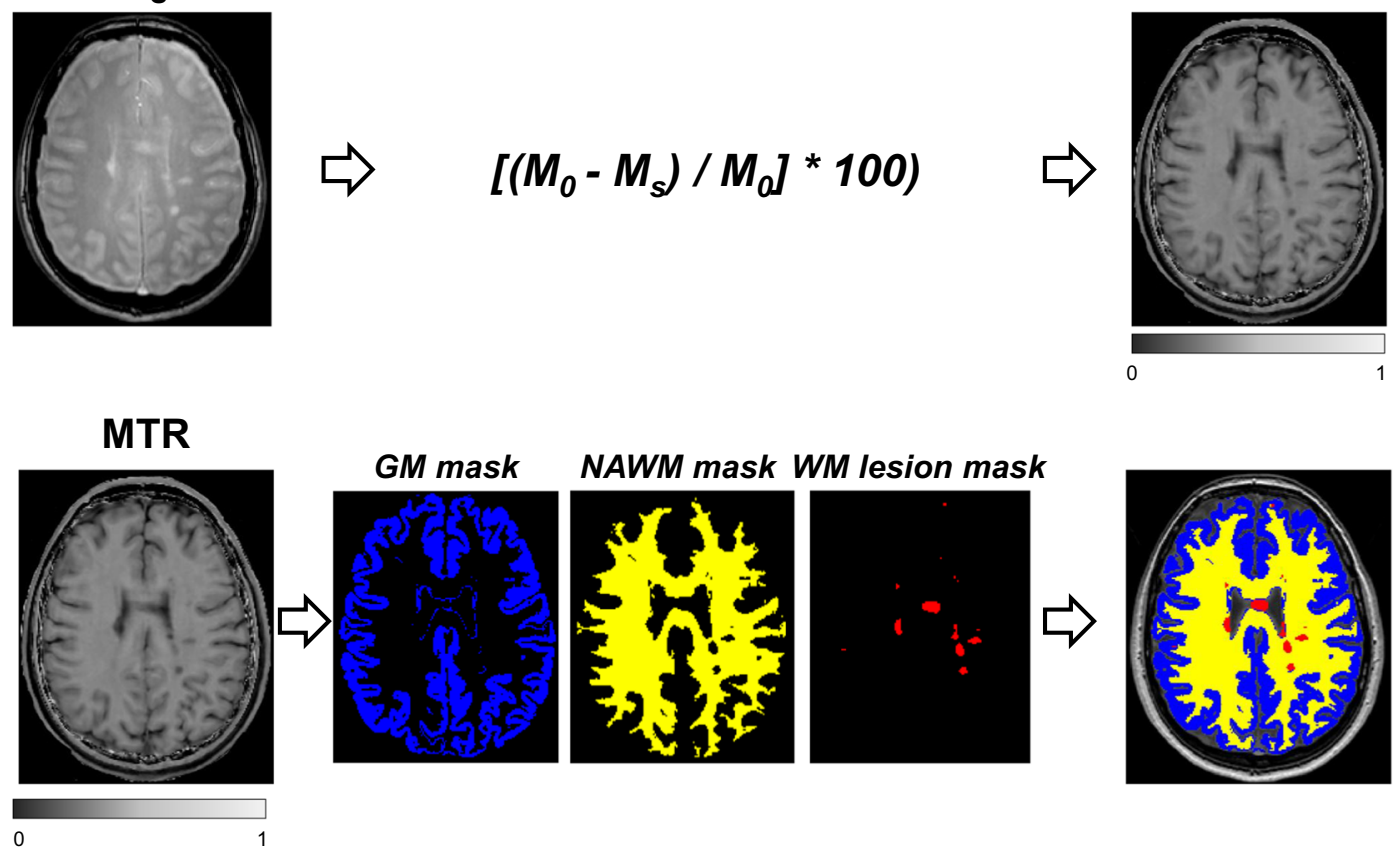

Fig. 2 Schematic representation of the pipeline applied to quantify T1w/ T2-w ratio and MTR in WM lesions, NAWM and GM. (a) To obtain T1w/T2-w ratio, native T1- and T2-weighted sequences underwent intensity bias correction, then calibration adjusting the intensity histograms using the lowest and highest intensity peaks derived from the ocular and temporal muscles masks on T1- and T2-weighted images. Finally, T2-weighted sequences were co-registered on T1-weighted images and the ratio between these images (i.e., T1w/T2w-ratio) was calculated. (b)

MTR images at each timepoint were calculated according to the formula: $\mathrm{MTR}=(\mathrm{M} 0$ [nonsaturated] $-\mathrm{MS}$ [saturated] $) / \mathrm{M} 0 \times 100) .(\mathrm{c}) \mathrm{T} 1 \mathrm{w} / \mathrm{T} 2 \mathrm{w}-$ ratio and MTR values within WM lesions, NAWM, and GM were derived. Abbreviations: $\mathrm{GM}=$ gray matter; $\mathrm{M} 0=$ nonsaturated image; $\mathrm{MS}=$ saturated image, $\mathrm{MTR}=$ magnetization transfer ratio; NAWM = normal-appearing white matter; $\mathrm{T} 1 \mathrm{w}=\mathrm{T} 1$-weighted; $\mathrm{T} 2 \mathrm{w}=\mathrm{T} 2$-weighted; $\mathrm{WM}=$ white matter 
T1w/T2w-ratio values within the GM and NAWM were derived by imposing the previously obtained tissue segmentation masks on the T1w/T2w-ratio image (Fig. 2).

The transformation estimated by co-registering T1- and T2-weighted images was applied to the WM lesion masks in order to extract $\mathrm{T} 1 \mathrm{w} / \mathrm{T} 2 \mathrm{w}$-ratio values also within WM lesions.

\section{MTR Quantification}

At each timepoint and for each subject, MTR images were calculated according to the formula: $\mathrm{MTR}=(\mathrm{M} 0$ [nonsaturated] $-\mathrm{MS}$ [saturated]) / M0 × 100) (Fig. 2).

T1- and T2-weighted images were rigidly co-registered on the MT reference image and the obtained transformations were applied to the GM, NAWM, and WM lesion masks. From the overlap between the co-registered masks and the MTR images, MTR values were obtained (Fig. 2).

\section{Statistical Analysis}

Comparisons of baseline demographic, clinical, and MRI measures were performed using Pearson chi-square, Fisher exact, Mann-Whitney, or two-sample $t$ tests as appropriate. T2-hyperintense LVs were log-transformed before analysis. We assessed within- and between-group longitudinal changes of continuous variables by linear mixed models, accounting for the repeated measures design with an unstructured variance-covariance matrix. Logistic and negative binomial models were used for dichotomous and count data. To adjust for baseline between-group imbalances, analyses were weighted by the inverse probability of treatment calculated using a logistic model including all baseline demographic and clinical characteristics with a standardized difference (according to Yang's and Dalton's method) $>0.10$, except for T2-hyperintense lesion number, strongly correlated with T2hyperintense LVs $($ rho $=0.78, p<0.001)$. $p$ values obtained from such analyses for $\mathrm{T} 1 \mathrm{w} / \mathrm{T} 2 \mathrm{w}$-ratio and MTR values underwent false discovery rate (Benjamini-Hochberg procedure) correction, to account for the overall number of within- and between-group comparisons tested, for each measure in each tissue (i.e., WM lesions, NAWM, and GM), separately. SAS software (version 9.4) was used for statistical analysis with a $p$ value $<0.05$ considered statistically significant.

\section{Results}

\section{Clinical and Conventional MRI Findings}

Table 1 summarizes clinical and conventional MRI findings in the two treatment groups. At baseline, fingolimod and natalizumab patients showed similar clinical and MRI characteristics. Four out of $30(13 \%)$ RRMS starting natalizumab were treatment naïve, 23/30 $(77 \%)$ were previously treated with first-line treatments (glatiramer acetate or interferon $\beta$ ), whereas $3 / 30(10 \%)$ received a second-line therapy or an immunosuppressant (two patients shifted from fingolimod, one from cyclophosphamide). Eighteen out of 25 (72\%) RRMS starting fingolimod were treated with first-line treatments (glatiramer acetate or interferon $\beta$ ), whereas $7 / 28$ (28\%) received a second-line therapy or an immunosuppressant (six patients shifted from natalizumab, one from cyclophosphamide).

At M24, both drugs significantly reduced the ARR compared to T0 $(p<0.001)$, without significant difference between-groups $(p=0.07)$. EDSS score remained stable similarly in both groups ( $p=0.67)$, without between-group differences in 3-month CDP ( $p=0.32)$.

Natalizumab patients had a significantly lower number and prevalence of new T2-hyperintense compared to fingolimod ( $p=0.03$ and 0.04 , respectively). T2hyperintense LV increased in fingolimod group $(p<0.001)$ and decreased in natalizumab group $(p=$ $0.001)$, whereas the prevalence of Gd-enhancing lesions was similar between treatment groups $(p=0.43)$.

\section{T1w/T2w-Ratio Findings}

At T0, GM, NAWM, and WM lesion T1w/T2w-ratio values were not significantly different between the two treatment groups ( $p \geq 0.32$ ) (Fig. 3 and Supplementary Table 1).

Figure 3 and Supplementary Table 1 show the longitudinal T1w/T2w-ratio changes in the different tissue types.

In WM lesions, T1w/T2w-ratio increased significantly at M12 vs. T0 $(p=0.01)$ and then remained stable at M24 in fingolimod group, whereas T1w/T2w-ratio increased significantly at M6 vs. T0 $(p=0.035)$ and then decreased significantly at M24 vs. M6 and M12 in natalizumab group $(p=0.001$ and 0.009 , respectively, the former surviving FDR-correction). No between-group differences were found.

In NAWM, T1w/T2w-ratio remained stable over 2 years in the natalizumab group, whereas a significant increase was found in the fingolimod group at M12 and M24 vs. M6 ( $p=0.002$ and 0.003 , both surviving FDR correction). No between-group differences were found.

In GM, T1w/T2w-ratio showed no significant changes over 2 years in the natalizumab group, whereas the fingolimod group showed a significant lower T1w/T2wratio at M6 vs. T0 $(p=0.014)$, followed by a gradual T1w/T2w-ratio increase that was significant at M24 vs. T0 $(p=0.008)$ and significantly different between groups $(p=0.034)$. 
Table 1 Main demographic, clinical, and conventional MRI findings at baseline and during the follow-up in relapsing-remitting multiple sclerosis patients starting fingolimod or natalizumab

\begin{tabular}{|c|c|c|c|c|}
\hline \multirow{2}{*}{$\begin{array}{l}\text { Variable } \\
\text { Baseline findings }\end{array}$} & \multirow{2}{*}{$\begin{array}{l}\text { Fingolimod } \\
(n=25)\end{array}$} & \multirow{2}{*}{$\begin{array}{l}\text { Natalizumab } \\
(n=30)\end{array}$} & \multicolumn{2}{|c|}{ Fingolimod vs natalizumab } \\
\hline & & & St. diff. & $p$ \\
\hline Women/men (\%) & $15(60) / 10(40)$ & $18(60) / 12(40)$ & 0 & $0.99^{\mathrm{a}}$ \\
\hline Mean age (SD) (year) & $37.5(8.7)$ & $36.8(10.2)$ & 0.07 & $0.81^{\mathrm{b}}$ \\
\hline Mean disease duration (SD) (years) & $11.1(6.6)$ & $9.5(6.8)$ & 0.25 & $0.36^{\mathrm{b}}$ \\
\hline Median ARR in the previous year (IQR) & $1(0 ; 1)$ & $1(1 ; 2)$ & 0.25 & $0.48^{\mathrm{d}}$ \\
\hline Last DMT\# (\%): none/1st line/2nd line & $0(0) / 18(72) / 7(28)$ & $4(13) / 23(77) / 3(10)$ & 0.70 & $0.06^{\mathrm{d}}$ \\
\hline Median EDSS (IQR) & $2.0(1.5 ; 3.0)$ & $2.0(1.5 ; 4.0)$ & 0.20 & $0.46^{\mathrm{c}}$ \\
\hline Median T2-hyperintense lesion number (IQR) & $\begin{array}{l}75 \\
(40 ; 114)\end{array}$ & $\begin{array}{l}53 \\
(31 ; 90)\end{array}$ & 0.38 & $0.17^{\mathrm{c}}$ \\
\hline Median T2-hyperintense LV (ml) (IQR) & $6.3(2.9 ; 12.0)$ & $5.1(2.1 ; 13.0)$ & 0.16 & $0.54^{\mathrm{b}}$ \\
\hline Median Gd-enhancing lesion number (IQR) & $0(0 ; 0)$ & $0(0 ; 1)$ & 0.43 & $0.40^{\mathrm{d}}$ \\
\hline Longitudinal findings & & & Adjustec & \\
\hline Median ARR (IQR) & $0.0(0.0 ; 0.5)^{\circ}$ & $0.00(0.00 ; 0.00)^{\circ}$ & 0.07 & \\
\hline Number $(\%)$ of patients with relapse(s) & $6(24 \%)$ & $1(3 \%)$ & 0.06 & \\
\hline Median EDSS change (IQR) & $0.0(-0.5 ; 0.0)$ & $0.0(-0.5 ; 0.0)$ & 0.67 & \\
\hline Number $(\%)$ of patients with 3-month CDP & $0(0 \%)$ & $2(7 \%)$ & 0.32 & \\
\hline Median number of new T2-hyperintense lesions (IQR) & $1(0 ; 2)$ & $0(0 ; 1)$ & 0.03 & \\
\hline Number $(\%)$ of patients with new T2-hyperintense lesions & $16(64 \%)$ & $10(33 \%)$ & 0.04 & \\
\hline Number $(\%)$ of patients with $\geq 1$ Gd-enhancing lesion during the follow-up & $2(8 \%)$ & $0(0 \%)$ & 0.43 & \\
\hline Median T2-hyperintense LV change (ml) (IQR) & $1.27(0.36 ; 2.62)^{*}$ & $-0.21(-1.00 ; 0.01)^{*}$ & $<0.001$ & \\
\hline
\end{tabular}

\# = first line = immunomodulants (i.e., glatiramer acetate and interferon $\beta$ ); second line = cyclophosphamide, fingolimod, or natalizumab. For the fingolimod group: six patients shifted from natalizumab, one from cyclophosphamide; for the natalizumab group: two patients shifted from fingolimod, one from cyclophosphamide

${ }^{\circ}$ Significant change compared to the ARR of the year before treatment initiation $(\mathrm{p}<0.001)$

*Significant change within the group vs. M0 $(p \leq 0.001)$

${ }^{\mathrm{a}}$ Chi-square test; ${ }^{\mathrm{b}}$ two-sample $t$ test; ${ }^{\mathrm{c}}$ Mann-Whitney test; ${ }^{\mathrm{d}}$ Fisher exact test; ${ }^{\mathrm{e}} p$ values from inverse probability of treatment-weighted generalized linear models, correcting for all baseline demographic and clinical characteristics showing a standardized difference $>0.10$ between groups: disease duration, annualized relapse rate in the previous year, last treatment, EDSS, T2-hyperintense LV and number of Gd-enhancing lesions

Statistically significant comparisons are shown in italics

Abbreviations: $A R R$, annualized relapse rate; EDSS, Expanded Disability Status Scale; $G d$ gadolinium; IQR, interquartile range; $L V$, lesion volume; $m l$, milliliter; $M R I$, magnetic resonance imaging; RRMS, relapsing-remitting multiple sclerosis; $S D$, standard deviation; St. Diff., standardized difference

\section{MTR Findings}

At T0, GM, NAWM, and WM lesion MTR was not significantly different between the two treatment groups $(p \geq 0.29)$ (Fig. 4.

Figure 4 and Supplementary Table 1 show the longitudinal MTR changes in the different tissue types.

In WM lesions, MTR gradually increased over 2 years in the fingolimod group, being significantly higher at M6, M12, and M24 vs. T0 $(p=0.008,0.027$, and 0.001 , the last surviving FDR correction) and at M24 vs. M6 and M12 ( $p=0.048$ and 0.039). In the natalizumab group, WM lesion MTR increased significantly at M6 vs. T0 $(p=0.028)$, decreased significantly at M12 vs. M6 $(p=0.037)$, and then remained stable at
M24. A significant between-group difference was found at M24 vs. M6 $(p=0.014)$.

In NAWM, MTR remained stable over 2 years in the natalizumab group, whereas a significant increase was found in the fingolimod group at M24 vs. M12 ( $p=0.001$, surviving FDR-correction), with a significant between-group difference ( $p=0.005$, surviving FDR correction).

In GM, MTR showed no significant changes over 2 years in the natalizumab group, whereas MTR was significantly higher at M24 vs. all the previous timepoints (vs. T0 $p=0.002$, vs. M6 $p=0.017$, vs. M12 $p=0.003$, with comparisons between T0 and M12 surviving FDR correction), with a significant between-group difference at M24 vs. M6 $(p=0.033)$ and at M24 vs. M12 $(p=0.020)$. 


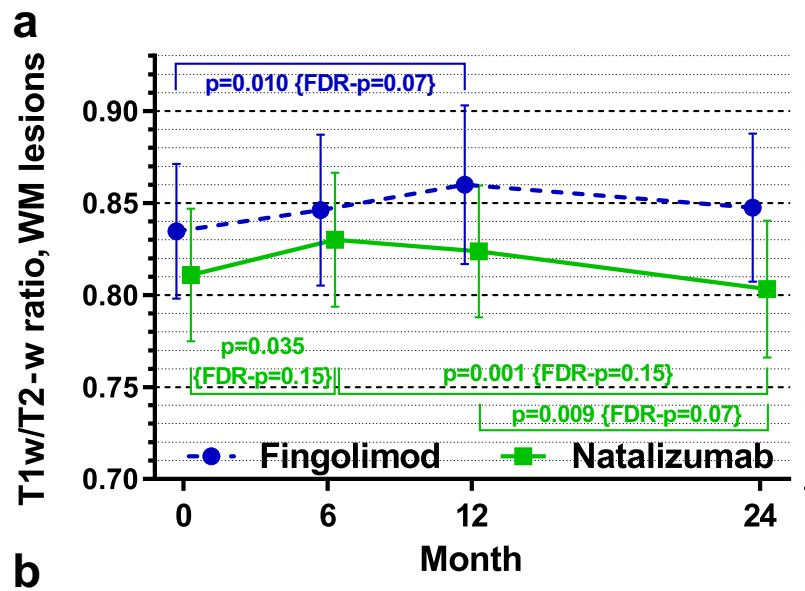

\begin{tabular}{cccc} 
& Fingolimod & Natalizumab & $\begin{array}{c}\text { Between-group } \\
\text { difference } \mathbf{9 5 \%} \text { CI) }\end{array}$ \\
\cline { 2 - 4 } & \multicolumn{2}{c}{ Estimated mean difference } & [p] \\
\hline M6 vs & $0.01(-0.00 ; 0.03)$ & $0.02(0.00 ; 0.04)$ & $-0.01(-0.03 ; 0.02)$ \\
T0 & {$[0.16]$} & {$[\mathbf{0 . 0 3 5}]\{0.15\}$} & {$[0.52]$} \\
\hline M12 vs & $0.03(0.01 ; 0.04)$ & $0.01(-0.01 ; 0.03)$ & $0.01(-0.02 ; 0.04)$ \\
T0 & {$[\mathbf{0 . 0 1 0}\{0.07\}$} & {$[0.20]$} & {$[0.37]$} \\
\hline M24 vs & $0.00(-0.01 ; 0.02)$ & $-0.03(-0.04 ;-0.01)$ & $0.03(0.01 ; 0.05)$ \\
M6 & {$[0.87]$} & {$[\mathbf{0 . 0 0 1}]\{\mathbf{0 . 0 2 5}\}$} & {$[\mathbf{0 . 0 1 5}\{0.08\}$} \\
\hline M24 vs & $-0.01(-0.03 ; 0.00)$ & $-0.02(-0.04 ;-0.01)$ & $0.01(-0.01 ; 0.03)$ \\
M12 & {$[0.06]$} & {$[\mathbf{0 . 0 0 9}\{0.07\}$} & {$[0.43]$} \\
\hline & & &
\end{tabular}

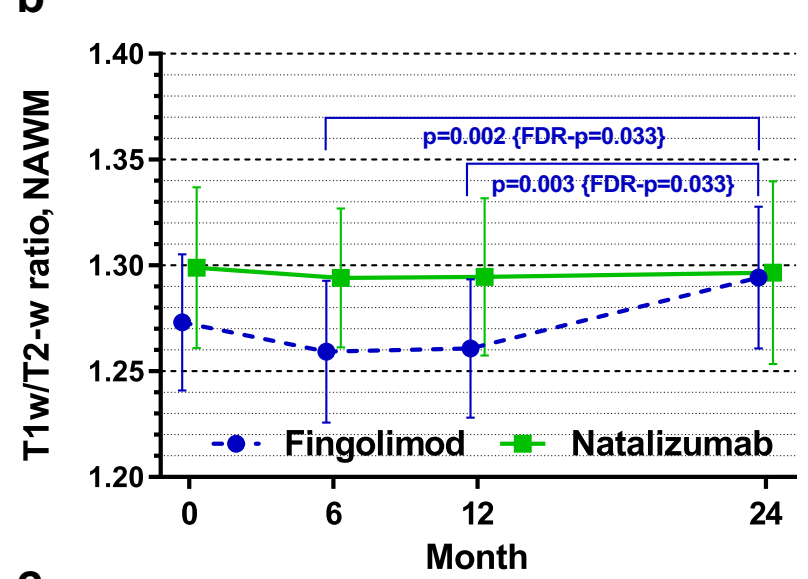

\begin{tabular}{cccc} 
& Fingolimod & Natalizumab & $\begin{array}{c}\text { Between-group } \\
\text { difference }(\mathbf{9 5 \%} \mathbf{C l})\end{array}$ \\
\cline { 2 - 4 } & \multicolumn{2}{c}{ Estimated mean difference } & [p] \\
\hline M24 vs & $0.03(0.01 ; 0.06)$ & $0.00(-0.02 ; 0.03)$ & $0.03(-0.00 ; 0.07)$ \\
M6 & {$[\mathbf{0 . 0 0 2}]\{\mathbf{0 . 0 3 3}\}$} & {$[0.85]$} & {$[0.07]$} \\
\hline M24 vs & $0.03(0.01 ; 0.05)$ & $0.00(-0.02 ; 0.03)$ & $0.03(-0.00 ; 0.06)$ \\
M12 & {$[\mathbf{0 . 0 0 3}]\{\mathbf{0 . 0 3 3}\}$} & {$[0.87]$} & {$[0.06]$} \\
\hline
\end{tabular}

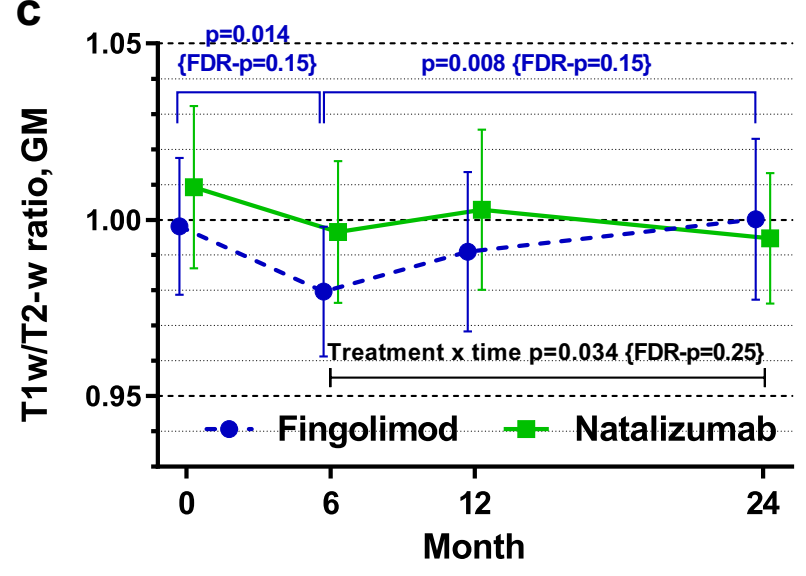

Fig. 3 Longitudinal T1w/T2-w ratio values in WM lesions, NAWM, and GM in RRMS starting fingolimod or natalizumab. Evolution of T1w/T2w ratio values of (a) WM lesions, (a) NAWM, and (c) GM. The corresponding significant results are also shown. Comparisons were performed with inverse probability of treatment-weighted linear mixed models, correcting for all baseline demographic and clinical characteristics showing a standardized difference $>0.10$ between groups: disease duration, annualized relapse rate in the previous year, last treatment, EDSS, T2-hyperintense LV, and number of Gd-enhancing lesions. Abbreviations: $\mathrm{CI}=$ confidence interval; $\mathrm{FDR}=$ false discovery rate; $\mathrm{GM}=$ gray matter; $\mathrm{M}=$ month; $\mathrm{NAWM}=$ normal-appearing white matter; $\mathrm{T} 1 \mathrm{w}=\mathrm{T} 1$-weighted; $\mathrm{T} 2 \mathrm{w}=\mathrm{T} 2$-weighted; $\mathrm{WM}=$ white matter

\section{Discussion}

By evaluating two semi-quantitative MRI measures sensitive to the different pathological substrates of MS, our study allowed us to compare the dynamic changes of T1w/T2wratio and MTR in both focal WM lesions and NA tissues during the first 2 years of treatment with natalizumab and fingolimod in patients with RRMS.

Our results suggested that these drugs may promote an early and effective resolution of inflammation, exert reparative processes in focal WM lesions, and promote neuroprotective effects in NA tissues. 


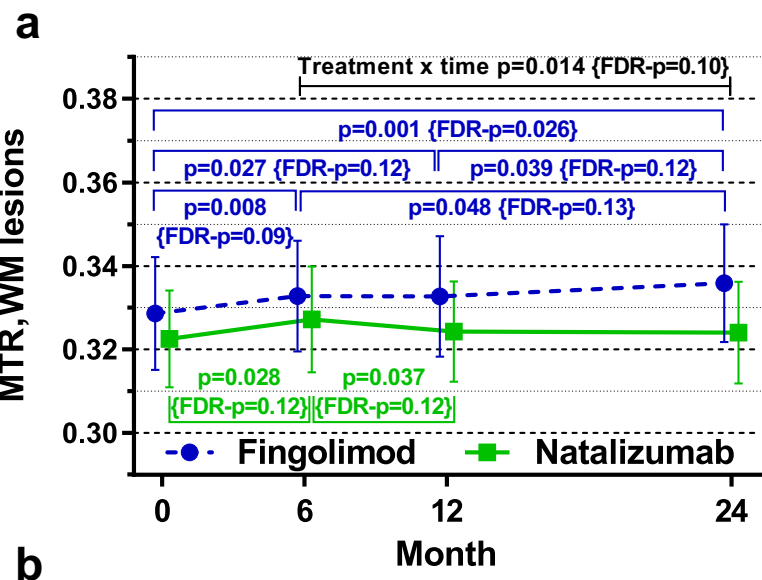

\begin{tabular}{cccc} 
& Fingolimod & Natalizumab & $\begin{array}{c}\text { Between-group } \\
\text { difference } \mathbf{( 9 5 \% ~ C I )}\end{array}$ \\
\cline { 2 - 4 } & \multicolumn{2}{c}{ Estimated mean difference } & {$[\mathbf{p}]$} \\
\hline M6 vs & $0.004(0.001 ; 0.007)$ & $0.005(0.001 ; 0.009)$ & $-0.001(-0.006 ; 0.005)$ \\
T0 & {$[\mathbf{0 . 0 0 8}[\{0.09\}$} & {$[\mathbf{0 . 0 2 8}[\{0.12\}$} & {$[0.84]$} \\
\hline M12 vs & $0.004(0.000 ; 0.008)$ & $0.002(-0.002 ; 0.006)$ & $0.002(-0.003 ; 0.007)$ \\
T0 & {$[\mathbf{0 . 0 2 7}]\{0.12\}$} & {$[0.32]$} & {$[0.41]$} \\
\hline M24 vs & $0.007(0.003 ; 0.011)$ & $0.002(-0.003 ; 0.006)$ & $0.006(-0.001 ; 0.012)$ \\
M0 & {$[\mathbf{0 . 0 0 1}\{\mathbf{0 . 0 2 6}\}$} & {$[0.52]$} & {$[0.08]$} \\
\hline M12 vs & $-0.000(-0.002 ; 0.002)$ & $-0.003(-0.005 ;-0.000)$ & $0.003(-0.001 ; 0.006)$ \\
M6 & {$[0.94]$} & {$[\mathbf{0 . 0 3 7}]\{0.12\}$} & {$[0.12]$} \\
\hline M24 vs & $0.003(0.000 ; 0.006)$ & $-0.003(-0.007 ; 0.001)$ & $0.006(0.001 ; 0.011)$ \\
M6 & {$[\mathbf{0 . 0 4 8}\{\{0.13\}$} & {$[0.11]$} & {$[\mathbf{0 . 0 1 4}]\{0.10\}$} \\
\hline M24 vs & $0.003(0.000 ; 0.006)$ & $-0.000(-0.004 ; 0.003)$ & $0.004(-0.001 ; 0.008)$ \\
M12 & {$[\mathbf{0 . 0 3 9}\{0.12\}$} & {$[0.85]$} & {$[0.14]$} \\
\hline & & &
\end{tabular}

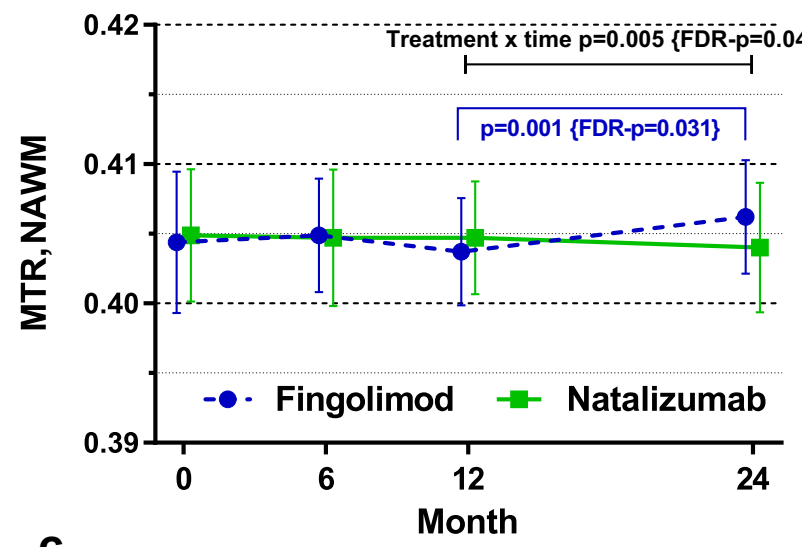

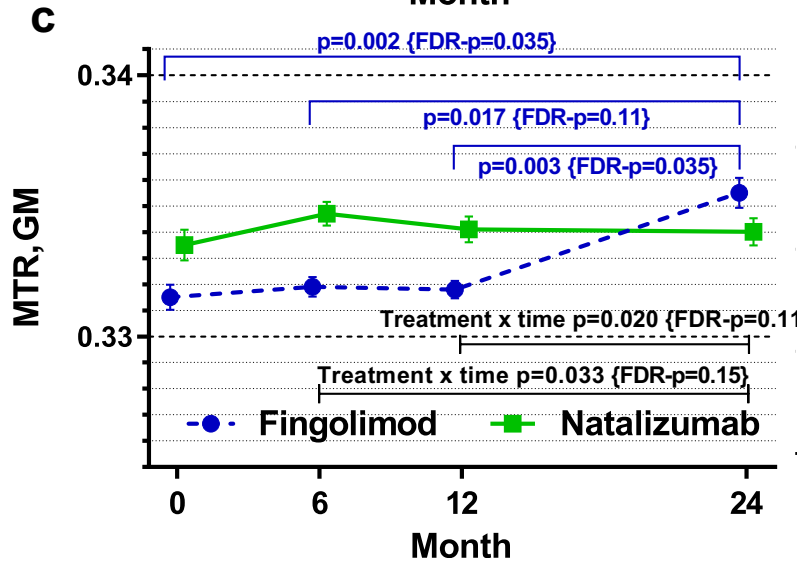

Fig. 4 Longitudinal MTR values in WM lesions, NAWM and GM in RRMS starting fingolimod or natalizumab. Evolution of MTR values of (a) WM lesions, (b) NAWM, and (c) GM. The corresponding significant results are also shown. Comparisons were performed with inverse probability of treatment-weighted linear mixed models, correcting for all baseline demographic and clinical characteristics showing a standardized difference $>0.10$ between groups: disease duration, annualized relapse rate in the previous year, last treatment, EDSS, T2-hyperintense LV, and number of Gd-enhancing lesions. Abbreviations: $\mathrm{CI}=$ confidence interval; $\mathrm{FDR}=$ false discovery rate; $\mathrm{GM}=$ gray matter; $\mathrm{M}=$ month; MTR = magnetization transfer ratio; NAWM = normal-appearing white matter; $\mathrm{WM}=$ white matter
The analysis of focal WM lesions revealed that, during the first 6 months, both treatment groups showed a significant increase of T1w/T2w-ratio and MTR values. This is expected since both drugs limit significantly and quickly lymphocyte migration into the CNS and promote a recovery of blood-brain barrier damage [27-29]. These findings suggest that natalizumab and fingolimod exert a similar, early and effective reduction of edema and acute inflammation. Our results are consistent with previous studies evaluating lesional MTR changes with highly effective anti-inflammatory treatments, including interferon beta- $1 \mathrm{~b}$, natalizumab, and hematopoietic stem cell transplantation $[5-8,30]$, as well as with positron emission tomography (PET) studies demonstrating that both fingolimod and natalizumab promote a significant 
reduction of activated microglia in focal WM lesions after 612 months of treatment [31-33].

Noteworthy, the increase of T1w/T2w-ratio and MTR continued several months after treatment start, thus after the resolution of inflammation. This evidence suggests that other mechanisms are likely to contribute, at least partially, to these findings. In our study, the positive effects seem more evident with fingolimod, since, in these patients, WM lesion T1w/ T2w-ratio increased gradually in the first year and then remained stable in the second year, whereas MTR gradually increased over the 2 years.

The prevention of further inflammatory events and the creation of a more favorable environment could contribute to enhance tissue recovery and remyelination in focal WM lesions [27-29]. Moreover, our findings are in line with experimental studies suggesting that fingolimod could protect against ongoing demyelinating processes also independently from its anti-inflammatory action and behind a preserved blood-brain barrier [27]. Fingolimod could also enhance remyelination by promoting the recruitment, differentiation, and axonal ensheathment capacity of oligodendrocyte precursor cells in demyelinating regions [27]. These effects seem less evident with natalizumab. This treatment downregulates significantly intrathecal activity of innate ad adaptive immune system but does not exert direct effect in CNS resident cells [28, 29]. Accordingly, the early recovery of lesional T1w/ T2w-ratio and MTR in natalizumab patients could reflect a resolution of inflammation and a more limited remyelinating capacity. Subsequently, these reparative mechanisms are counterbalanced by ongoing demyelination and neuroaxonal loss that are still likely to occur at a low rate during subsequent timepoints. However, these detrimental processes could also be attributable, at least partially, to the accumulation of structural damage associated with disease activity that occurred before treatment start.

Other interesting results are those derived from the evaluation of T1w/T2w-ratio and MTR in NAWM and GM. Pathological studies have demonstrated that, compared to lesions, NAWM and GM are characterized by a milder degree of perivascular inflammatory infiltrates, edema, diffuse microglia activation, diffuse neuro-axonal injury, and gliosis $[34,35]$. These processes are secondary to neuro-axonal damage within focal lesions but can also develop independently from lesions [34, 35] and contribute to clinical disability [36].

Our study showed that fingolimod is associated with a significant increase of NAWM T1w/T2w-ratio, NAWM MTR, and GM MTR. We also detected a decline followed by an increase of GM T1w/T2w-ratio.

These findings are in line with a recent study showing that after 1 year, fingolimod promoted an increase of fractional anisotropy and a decrease of radial diffusivity in the corticospinal tracts and in the superior and inferior cerebellar peduncles in RRMS patients with piramidal or cerebellar impairment, respectively [16]. It is noteworthy that, similarly to WM lesions, increases of T1w/T2w-ratio and MTR occur especially in the second year of treatment. This suggests again that other neuroprotective processes beyond the resolution of acute inflammation may explain our findings. Experimental studies showed that fingolimod can exert beneficial effects on different resident CNS cells [27]. Fingolimod reduces the proinflammatory state and upregulates brain-derived neurotrophic factor (BDNF) production of astrocytes, and promotes recruitment and activation of oligodendrocyte precursor cells [27]. Moreover, fingolimod downregulates the expression of pro-inflammatory cytokines and upregulates the production of several neurotrophic factors in microglia [27].

Interestingly, we also found that natalizumab stabilized both NAWM and GM microarchitecture over 2 years. Our findings are in line with a diffusion tensor MRI study showing that NAWM diffusivity metrics were stable for 4 years during natalizumab treatment [18] and with a PET study showing a stability of activated microglia in NAWM and GM after 6 months of treatment [31]. Conversely, they seem in contrast with other studies showing a recovery of MTR or diffusivity measures in NA brain tissues over 1 or 2 years $[6,10,17]$ and a reduction of activated microglia in NAWM after 1 year of treatment [33]. The small sample sizes of our groups, heterogeneities in MS patients' characteristics, and methods of analyses (e.g., MTR vs. diffusion tensor MRI; global or regional evaluations) may contribute to explain discrepancies with other studies. It is also likely that the differences among studies could be due to the co-occurrence, during treatment, of pathological processes that influence MRI measures in the opposite direction, such as concomitant demyelination and remyelination, secondary neuro-axonal damage but also enhanced neuro-axonal integrity, and reduction of activated microglia and gliosis.

Clearly, our results should be interpreted carefully and cautiously, since our study has some limitations. Two relatively small cohorts of RRMS were recruited without randomization. However, treatments were chosen according to real-life clinical guidelines and the acquisition of MRI exams at specific scheduled timepoints using the same scanner is challenging in the clinical setting. To limit the influence of baseline differences, the analyses were adjusted for unbalanced baseline findings. Moreover, several comparisons did not survive FDR correction. Accordingly, further studies, with larger cohorts of RRMS patients starting natalizumab or fingolimod are needed to confirm our findings. The availability of a group of healthy controls and of another one with untreated MS patients could have further supported the potential beneficial effects of fingolimod and natalizumab on the microstructure of both brain WM lesions and NA tissues. In particular, the evaluation of healthy controls would have contributed to better understand the severity and the longitudinal changes of microstructural tissue damage in RRMS patients starting 
either natalizumab or fingolimod. Moreover, the assessment of scan-rescan variability of both $\mathrm{T} 1 \mathrm{w} / \mathrm{T} 2-\mathrm{w}$ ratio and MTR could be relevant to demonstrate the reliability of these two measures. Unfortunately, scan-rescan assessment of these measures was not available; however, previous studies suggested that both $\mathrm{T} 1 \mathrm{w} / \mathrm{T} 2 \mathrm{w}$ ratio and MTR have a good intraand inter-subject reproducibility [25,37]. The availability of another group of untreated MS patients could have allowed to better evaluate the beneficial effects of these treatments on progressive damage due to MS.

Although a slice thickness of $4 \mathrm{~mm}$ for the MTR acquisition could limit a precise estimation of MTR values, it is noteworthy that we obtained an averaged measure for the whole WM lesions, NAWM, and GM, thus mitigating the possible "data loss" due to the image resolution along the $z$ axis.

Finally, a longer follow-up could be rewarding to better investigate the beneficial effects of both drugs over time.

In conclusion, together with a significant prevention of further clinical and MRI disease activity and a stabilization of clinical disability, our study suggests that natalizumab could promote an early recovery of lesional damage and prevent microstructural damage accumulation in NAWM and GM during the first 2 years of treatment. Fingolimod could enhance tissue damage recovery being visible after 6 months in lesions and after 2 years in NA brain tissues.

Although the limited sample size and the lack of a randomization did not allow to provide specific suggestions regarding how and to whom to prescribe natalizumab rather than fingolimod, it is noteworthy that both drugs exerted positive neuroprotective effects on different tissue types, thus promoting their use for RRMS patients following regulations and real-life clinical guidelines.

Required Author Forms Disclosure forms provided by the authors are available with the online version of this article.

Supplementary Information The online version contains supplementary material available at https://doi.org/10.1007/s13311-020-00997-1.

\section{Compliance with Ethical Standards}

Conflict of Interest The authors declare that they have no competing interests in relation to this work. Potential conflicts of interest outside the submitted work are as follows.

P. Preziosa received speaker honoraria from Biogen Idec, Novartis, Merck Serono, and ExceMED.

L. Storelli, A. Meani and M. Rodegher have nothing to disclose.

L. Moiola has received personal compensation for consulting, serving on a scientific advisory board, speaking, or other activities with SanofiGenzyme, Novartis, Teva, Merck-Serono, Biogen, Roche, Excemed.

M. Filippi is Editor-in-Chief of the Journal of Neurology; received compensation for consulting services and/or speaking activities from Bayer, Biogen Idec, Merck-Serono, Novartis, Roche, Sanofi Genzyme, Takeda, and Teva Pharmaceutical Industries; and receives research support from Biogen Idec, Merck-Serono, Novartis, Roche, Teva Pharmaceutical Industries, Italian Ministry of Health, Fondazione Italiana Sclerosi Multipla, and ARiSLA (Fondazione Italiana di Ricerca per la SLA).

M.A. Rocca received speaker honoraria from Biogen Idec, Novartis, Genzyme, Teva, Merck Serono, Roche, Celgene, and Bayer and receives research support from the Italian Ministry of Health, MS Society of Canada and Fondazione Italiana Sclerosi Multipla.

\section{References}

1. Filippi M, Bruck W, Chard D, Fazekas F, Geurts JJG, Enzinger C, et al. Association between pathological and MRI findings in multiple sclerosis. Lancet Neurol. 2019;18(2):198-210.

2. Schmierer K, Scaravilli F, Altmann DR, Barker GJ, Miller DH. Magnetization transfer ratio and myelin in postmortem multiple sclerosis brain. Ann Neurol. 2004;56(3):407-15.

3. Moccia M, van de Pavert S, Eshaghi A, Haider L, Pichat J, Yiannakas M, et al. Pathological correlates of the magnetization transfer ratio in multiple sclerosis. Neurology. 2020.

4. Schmierer K, Parkes HG, So PW, An SF, Brandner S, Ordidge RJ, et al. High field (9.4 Tesla) magnetic resonance imaging of cortical grey matter lesions in multiple sclerosis. Brain. 2010;133(Pt 3): 858-67.

5. Rocca MA, Preziosa P, Filippi M. Application of advanced MRI techniques to monitor pharmacologic and rehabilitative treatment in multiple sclerosis: current status and future perspectives. Expert Rev Neurother. 2019;19(9):835-66.

6. Zivadinov R, Dwyer MG, Hussein S, Carl E, Kennedy C, Andrews $\mathrm{M}$, et al. Voxel-wise magnetization transfer imaging study of effects of natalizumab and IFNbeta-1a in multiple sclerosis. Mult Scler. 2012;18(8):1125-34.

7. Brown RA, Narayanan S, Arnold DL. Segmentation of magnetization transfer ratio lesions for longitudinal analysis of demyelination and remyelination in multiple sclerosis. Neuroimage. 2013;66:1039.

8. Chen JT, Collins DL, Atkins HL, Freedman MS, Arnold DL, Canadian MSBMTSG. Magnetization transfer ratio evolution with demyelination and remyelination in multiple sclerosis lesions. Ann Neurol. 2008;63(2):254-62.

9. Chen JT, Kuhlmann T, Jansen GH, Collins DL, Atkins HL, Freedman MS, et al. Voxel-based analysis of the evolution of magnetization transfer ratio to quantify remyelination and demyelination with histopathological validation in a multiple sclerosis lesion. Neuroimage. 2007;36(4):1152-8.

10. Romme Christensen J, Ratzer R, Bornsen L, Lyksborg M, Garde E, Dyrby TB, et al. Natalizumab in progressive MS: results of an openlabel, phase 2A, proof-of-concept trial. Neurology. 2014;82(17): 1499-507.

11. Button T, Altmann D, Tozer D, Dalton C, Hunter K, Compston A, et al. Magnetization transfer imaging in multiple sclerosis treated with alemtuzumab. Mult Scler. 2013;19(2):241-4.

12. Filippi M, Rocca MA, Pagani E, De Stefano N, Jeffery D, Kappos $\mathrm{L}$, et al. Placebo-controlled trial of oral laquinimod in multiple sclerosis: MRI evidence of an effect on brain tissue damage. $J$ Neurol Neurosurg Psychiatry. 2014;85(8):851-8.

13. Nakamura K, Chen JT, Ontaneda D, Fox RJ, Trapp BD. T1-/T2weighted ratio differs in demyelinated cortex in multiple sclerosis. Ann Neurol. 2017;82(4):635-9.

14. Righart R, Biberacher V, Jonkman LE, Klaver R, Schmidt P, Buck $\mathrm{D}$, et al. Cortical pathology in multiple sclerosis detected by the T1/ T2-weighted ratio from routine magnetic resonance imaging. Ann Neurol. 2017;82(4):519-29. 
15. Tsivgoulis G, Katsanos AH, Mavridis D, Grigoriadis N, Dardiotis E, Heliopoulos I, et al. The Efficacy of Natalizumab versus Fingolimod for Patients with Relapsing-Remitting Multiple Sclerosis: A Systematic Review, Indirect Evidence from Randomized Placebo-Controlled Trials and Meta-Analysis of Observational Head-to-Head Trials. PLoS One. 2016;11(9): e0163296.

16. Gurevich M, Waknin R, Stone E, Achiron A. Fingolimodimproved axonal and myelin integrity of white matter tracts associated with multiple sclerosis-related functional impairments. CNS Neurosci Ther. 2018;24(5):412-9.

17. Wiebenga OT, Schoonheim MM, Hulst HE, Nagtegaal GJ, Strijbis EM, Steenwijk MD, et al. White Matter Diffusion Changes during the First Year of Natalizumab Treatment in Relapsing-Remitting Multiple Sclerosis. AJNR Am J Neuroradiol. 2016;37(6):1030-7.

18. Ontaneda D, Sakaie K, Lin J, Wang XF, Lowe MJ, Phillips MD, et al. Measuring Brain Tissue Integrity during 4 Years Using Diffusion Tensor Imaging. AJNR Am J Neuroradiol. 2017;38(1): 31-8.

19. Fox RJ, Cronin T, Lin J, Wang X, Sakaie K, Ontaneda D, et al. Measuring myelin repair and axonal loss with diffusion tensor imaging. AJNR Am J Neuroradiol. 2011;32(1):85-91.

20. Preziosa P, Rocca MA, Riccitelli GC, Moiola L, Storelli L, Rodegher M, et al. Effects of Natalizumab and Fingolimod on Clinical, Cognitive, and Magnetic Resonance Imaging Measures in Multiple Sclerosis. Neurotherapeutics. 2020;17(1):208-17.

21. Preziosa P, Rocca MA, Pagani E, Storelli L, Rodegher M, Moiola $\mathrm{L}$, et al. Two-year regional grey and white matter volume changes with natalizumab and fingolimod. J Neurol Neurosurg Psychiatry. 2020;91(5):493-502.

22. Polman CH, O'Connor PW, Havrdova E, Hutchinson M, Kappos L, Miller DH, et al. A randomized, placebo-controlled trial of natalizumab for relapsing multiple sclerosis. $N$ Engl J Med. 2006;354(9):899-910.

23. Rudick RA, Stuart WH, Calabresi PA, Confavreux C, Galetta SL, Radue EW, et al. Natalizumab plus interferon beta-1a for relapsing multiple sclerosis. N Engl J Med. 2006;354(9):911-23.

24. Chard DT, Jackson JS, Miller DH, Wheeler-Kingshott CA. Reducing the impact of white matter lesions on automated measures of brain gray and white matter volumes. J Magn Reson Imaging. 2010;32(1):223-8.

25. Ganzetti M, Wenderoth N, Mantini D. Whole brain myelin mapping using T1- and T2-weighted MR imaging data. Front Hum Neurosci. 2014;8:671.

26. Tustison NJ, Avants BB, Cook PA, Zheng Y, Egan A, Yushkevich PA, et al. N4ITK: improved N3 bias correction. IEEE Trans Med Imaging. 2010;29(6):1310-20.
27. Hunter SF, Bowen JD, Reder AT. The Direct Effects of Fingolimod in the Central Nervous System: Implications for Relapsing Multiple Sclerosis. CNS Drugs. 2016;30(2):135-47.

28. Sellebjerg F, Cadavid D, Steiner D, Villar LM, Reynolds R, Mikol D. Exploring potential mechanisms of action of natalizumab in secondary progressive multiple sclerosis. Ther Adv Neurol Disord. 2016;9(1):31-43.

29. Stuve O, Marra CM, Bar-Or A, Niino M, Cravens PD, Cepok S, et al. Altered CD4+/CD8+ T-cell ratios in cerebrospinal fluid of natalizumab-treated patients with multiple sclerosis. Arch Neurol. 2006;63(10):1383-7.

30. Richert ND, Ostuni JL, Bash CN, Leist TP, McFarland HF, Frank JA. Interferon beta-1b and intravenous methylprednisolone promote lesion recovery in multiple sclerosis. Mult Scler. 2001;7(1): 49-58.

31. Kaunzner UW, Kang Y, Monohan E, Kothari PJ, Nealon N, Perumal J, et al. Reduction of PK11195 uptake observed in multiple sclerosis lesions after natalizumab initiation. Mult Scler Relat Disord. 2017;15:27-33.

32. Sucksdorff M, Rissanen E, Tuisku J, Nuutinen S, Paavilainen T, Rokka J, et al. Evaluation of the Effect of Fingolimod Treatment on Microglial Activation Using Serial PET Imaging in Multiple Sclerosis. J Nucl Med. 2017;58(10):1646-51.

33. Sucksdorff M, Tuisku J, Matilainen M, Vuorimaa A, Smith S, Keitila J, et al. Natalizumab treatment reduces microglial activation in the white matter of the MS brain. Neurol Neuroimmunol Neuroinflamm. 2019;6(4):e574.

34. Kutzelnigg A, Lucchinetti CF, Stadelmann C, Bruck W, Rauschka $\mathrm{H}$, Bergmann $\mathrm{M}$, et al. Cortical demyelination and diffuse white matter injury in multiple sclerosis. Brain. 2005;128(Pt 11):2705-12.

35. Haider L, Zrzavy T, Hametner S, Hoftberger R, Bagnato F, Grabner $\mathrm{G}$, et al. The topograpy of demyelination and neurodegeneration in the multiple sclerosis brain. Brain. 2016;139(Pt 3):807-15.

36. Filippi M, Preziosa P, Rocca MA. Microstructural MR Imaging Techniques in Multiple Sclerosis. Neuroimaging Clin N Am. 2017;27(2):313-33.

37. Inglese M, Horsfield MA, Filippi M. Scan-rescan variation of measures derived from brain magnetization transfer ratio histograms obtained in healthy volunteers by use of a semi-interleaved magnetization transfer sequence. AJNR Am J Neuroradiol. 2001;22(4): $681-4$.

Publisher's Note Springer Nature remains neutral with regard to jurisdictional claims in published maps and institutional affiliations. 